\title{
Barrier-Free Pedestrian Routing with Contraction Hierarchies
}

\author{
Ruoying Li \\ University of Konstanz \\ Comput. Inf. Sci. \\ Konstanz, Germany \\ ruoying.li@inf.uni- \\ konstanz.de
}

\author{
Adv.: Sabine Storandt \\ University of Konstanz \\ Comput. Inf. Sci. \\ Konstanz, Germany \\ storandt@inf.uni- \\ konstanz.de
}

\author{
Adv.: Uli Müller \\ geOps AG \\ Olten, Switzerland \\ uli.mueller@geops.ch
}

\author{
Adv.: David Weber \\ geOps $\mathrm{GmbH}$ \\ Freiburg, Germany \\ david.weber@geops.de
}

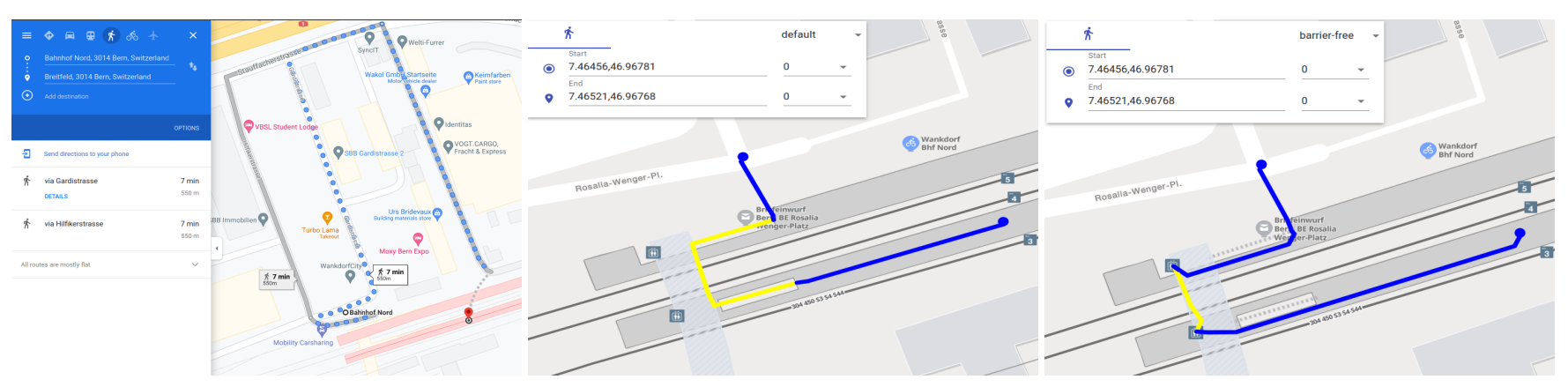

Figure 1: Pedestrian routing example at the train station of Bern, Switzerland. Google Maps (left) recommends a route with a huge detour that suggests hopping over train tracks to reach the destination. In the middle and right image, routes computed with our approach are shown. The route in the middle is the shortest route. It uses two stairs and a pedestrian bridge. The right route is the shortest barrier-free route and uses elevators to get from one platform to another.

\begin{abstract}
We present a holistic approach for pedestrian routing that allows computing shortest paths that may have indoor and outdoor sections. Such routes arise, for example, when the destination is not just an address but a specific store in a large mall, or when one needs to get to a certain track at a large train station. Currently, map services as Google Maps or OSRM do not offer such functionality. We identify and overcome three main challenges for answering such complex route planning queries: (i) Pedestrian routing requires fine-grained data, as the location of stairs and elevators, building entrances, building footprints, and elevation/level information. A single missing staircase can change the length of the computed path severely. (ii) Indoor routing has to be integrated carefully into classical path planning to allow the computation of sensible routes that may enter and exit buildings. (iii) Given the large amount of data to be considered in a query, acceleration techniques need to be applied in order to achieve interactive query times. Retrieving barrier-free routes for wheelchairs is also our important use case.
\end{abstract}

\section{CCS CONCEPTS}

\section{- Theory of computation $\rightarrow$ Shortest paths.}

Permission to make digital or hard copies of all or part of this work for personal or classroom use is granted without fee provided that copies are not made or distributed for profit or commercial advantage and that copies bear this notice and the full citation on the first page. Copyrights for components of this work owned by others than ACM must be honored. Abstracting with credit is permitted. To copy otherwise, or republish, to post on servers or to redistribute to lists, requires prior specific permission and/or a fee. Request permissions from permissions@acm.org.

SIGSPATIAL '21, November 2-5, 2021, Beijing, China

(c) 2021 Association for Computing Machinery.

ACM ISBN 978-1-4503-8664-7/21/11 . \$ \$15.00

https://doi.org/10.1145/3474717.3486797

\section{KEYWORDS}

GIS, pedestrian routing, indoor routing, shortest path

\section{ACM Reference Format:}

Ruoying Li, Adv.: Sabine Storandt, Adv.: Uli Müller, and Adv.: David Weber. 2021. Barrier-Free Pedestrian Routing with Contraction Hierarchies . In 29th International Conference on Advances in Geographic Information Systems (SIGSPATIAL '21), November 2-5, 2021, Beijing, China. ACM, New York, NY, USA, 2 pages. https://doi.org/10.1145/3474717.3486797

\section{INTRODUCTION}

Pedestrian routing is typically decoupled into outdoor routing along paths and pavements and indoor routing that allows navigating (multi-level) buildings. A plethora of algorithms have been developed for each scenario separately [3]. But there is a lack of scalable solution approaches that integrate both and allow to compute routes that traverse paths as well as buildings. Such routes are, however, of high practical relevance; e.g., for getting to destinations in complex buildings, as large train stations, airports, or university campuses. In Bern central train station, more than 90 stairs and elevators connect over 15 public areas. Finding a sensible route to a particular platform in such a large train station is non-trivial for passengers, and even more arduous if the path needs to be barrier-free. Map services as Google Maps do not support indoor routing yet. This leads to strange and sometimes even dangerous route recommendations as shown in Figure 1. OSRM has some indoor information but without floor levels or wheelchair accessibility, and sometimes also suggests routes that cross train tracks. Our goal is to design an integrated system for pedestrian route planning that recommends meaningful and safe routes. Everard's master thesis [1] provides an indoor/outdoor routing approach. However, it takes 17 hours to 
process the data of a single city. We optimize the data processing pipeline to enable pedestrian routing for a much larger area. Figure 1 shows such routes computed with our approach. A demo is available at https://pedestrian-routing-demo.geops.io.

\section{DATA EXTRACTION AND PROCESSING}

The first important step is to collect all necessary data for pedestrian routing. We fused data from OpenStreetMap (OSM) ${ }^{1}$ and station plans from Swiss federal railway $\mathrm{SBB}^{2}$ for that purpose, as there is not single data source that provides all required information.

From OSM, road and path networks were extracted as well as the outlines of public squares and areas. Based on the OSM labels, paths and areas are categorized into three classes: walkable, allowed, and avoid. For example, footways and public squares are classified as walkable; cycleways are classified as allowed, primary and secondary ways (large roads) are classified as avoid. While OSM occasionally contains some building information as entrance positions or the number of floors, this is not sufficiently fine-grained for our purpose. Therefore, we additionally use SBB train station plans. This data collection contains detailed footprint polygons for all large train stations in Switzerland, including floor information as well as floor connections as stairs and elevators.

\section{PEDESTRIAN ROUTING MODEL}

The second step is to construct a graph that models all feasible pedestrian routes, including ones that enter or cross buildings. To be able to compute meaningful routes later on, we also have to choose edge weights carefully.

Modeling Walkable Areas. To enable walking indoors or on platforms, all walkable polygons are covered with 8-connected grids. Since platforms often have a long strip shape, using an arbitrary grid orientation might lead to strange optimal route shapes. Therefore, grids were oriented parallel to the longest edge of the respective polygon, see Figure 2 for an illustration. The grids are first constructed with a fixed width for each polygon using the prime meridian and the equator as axes. Then, the angle from the equator to the longest edge of the polygon is determined and the new grid coordinates are derived with the help of a rotation matrix.

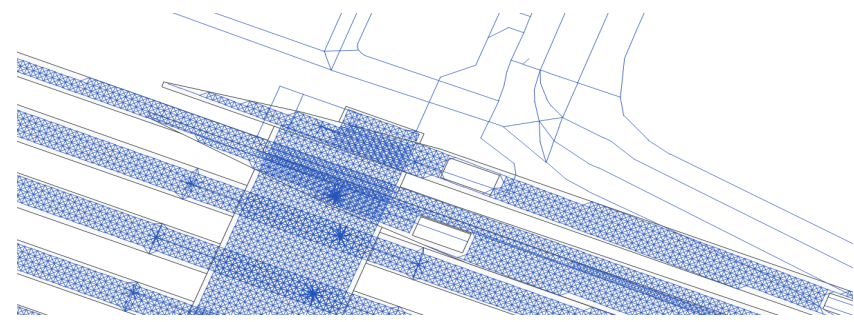

Figure 2: Train station and platforms with grid structure.

Connecting Paths and Areas. The walkable areas in the data set are typically disjoint and need to be connected to each other and the path network. Stair lines and elevator locations form the connections between the grid graphs of different floors in a building or to access pedestrian bridges. The stair line direction is derived from the object labels in the data collection. The elevator points

\footnotetext{
${ }^{1}$ https://www.openstreetmap.org/

${ }^{2}$ https://plans.trafimage.ch/
}

are connected to all reachable floors. Finally, indoor and area grids get connected to the path network based on entrance locations and path intersection points.

Choosing Edge Weights. For default pedestrian routing, edge costs are chosen proportional to the Euclidean length of the edge. Based on the category of the edge, these costs are modified by multiplication with a constant factor. This ensures that edges categorized as allowed or avoid are less likely to be chosen in a route.

To be able to compute barrier-free routes, we define a second cost value per edge. The barrier-free cost multiplier for an edge is huge if the edge is a stair or connected to stairs and low if it is the connection to an elevator or ramp. Otherwise, the costs are equal.

\section{EFFICIENT ROUTE COMPUTATION}

The constructed routing graph is significantly larger than the path network. Therefore, running plain Dijkstra's algorithm is too slow even for local queries. For accelerated shortest path planning in road networks, contraction hierarchies $(\mathrm{CH})$ [2] is one of the most widely used methods. It adds so-called shortcut edges to the graph that allow Dijkstra's algorithm to relax significantly fewer edges.

However, there are some peculiarities of $\mathrm{CH}$ on our combined path and grid network. First of all, we need to consider two weights for each edge. Secondly, $\mathrm{CH}$ adds too many shortcuts on the large grid structures of walkable areas where almost all nodes have a degree of eight. Therefore, we proceed in two phases: We first construct a shortcut set using Dijkstra's algorithm that allows to cross buildings. Then we construct $\mathrm{CH}$ on the path network and these shortcuts without considering grid nodes and edges. By assigning the nodes in the grids later on suitable $\mathrm{CH}$ attributes, we can still guarantee correct query answering between all node pairs. Our approach takes a fraction of the preprocessing time and space consumption than just applying $\mathrm{CH}$ to the whole graph.

\section{EXPERIMENTAL RESULTS}

We implement Dijkstra's algorithm and $\mathrm{CH}$ in $\mathrm{C}++$ and conducted experiments on an Intel(R) Xeon(R) Silver $4114 \mathrm{CPU}$ (clocked at 2.2 $\mathrm{GHz}$ ) with $100 \mathrm{~GB}$ of main memory. For benchmarking, we constructed the pedestrian routing graph for Switzerland, Germany, and Austria. The graph contains 169,307,710 nodes and 488,129,136 edges and is computed in about 10 hours using a single core. Among those, 25,365,823 nodes and 182,922,922 edges were added to the path network to enable walking through train stations or public areas. The preprocessing time to construct the $\mathrm{CH}$ graph was about 10 hours using four threads. On average, Dijkstra's algorithm took 67.108 seconds on the original graph but only 0.239 seconds on the $\mathrm{CH}$ graph with shortcuts. In conclusion, our integrated approach enables fast (barrier-free) pedestrian route planning.

Acknowledgements. We thank geOps AG for intense collaboration on this project.

\section{REFERENCES}

[1] Everad, B. Konzeption und Implementierung von Kombinierten Indoor/Outdoor Fußgängerrouting auf Basis von OSM- und CAD-Daten.

[2] Geisberger, R., Sanders, P., Schultes, D., and Vetter, C. Exact routing in large road networks using contraction hierarchies. Transportation Science 46, 3 (2012), 388-404.

[3] Liv, T., Li, H., Lu, H., Cheema, M. A., And Shou, L. Towards crowd-aware indoor path planning. Proc. VLDB Endow. 14, 8 (2021), 1365-1377. 